\title{
Development of Traditional Algorithm Used in Denoising to Enhance Contrast of the Color Images
}

\section{Hanan Hamid Ali Esraa Abd Al-Majed Asmaa Nadhim Rahma Saleem} hanan_hamidali@uomosul.edu.iq

College of Computer Sciences and Mathematics

University of Mosul, Mosul, Iraq

\section{Received on: 15/10/2012}

\section{ABSTRACT}

Accepted on: 30/01/2013

With the evolution of life and complexity there is a need to use the images in many scientific fields weather forecast, medical, engineering, and the fact that these images are a lot of distortion during capture due to the availability of cameras and Rate in abundance and in the hands of people who do not have enough experience in how to capture images in terms of quality lighting and distribution has become important use a variety of methods to improve these images show the best form of these methods and improve the image contrast (Contrast Enhancement).

In this research applied traditional algorithm filter to improve the Adaptive Contrast Enhancement Global Filter (ACE _mean) and has been the development of this algorithm to a new two Hybrid algorithms to improve the contrast Enhancement (Median Contrast Enhancement ACE_median) and the other algorithm is (Max \& Min Contrast Enhancement ACE_max\&min). the two hybrid algorithms obtained from merging two available methods, first is the traditional algorithm (ACE_mean) with the second methods of another practice in improving the image is (Smoothing Images Enhancement) which is used to removing the noise from images .the results of each method were compared with the results of other algorithms to show the best of them.

Keyword: Contrast, Adaptive Contrast Enhancement Global Filter.
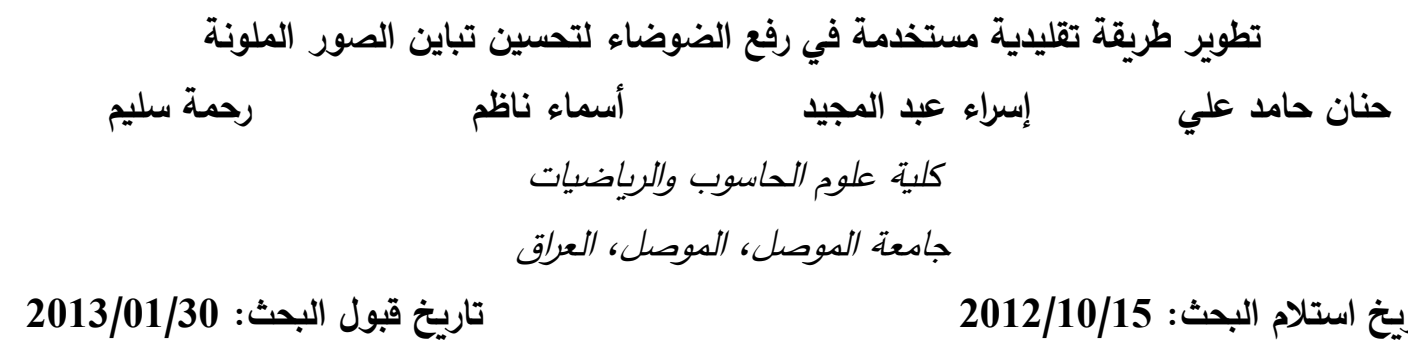

تاريخ استلام البحث: 2012/10/15

\section{الملخص}

مع تطور الحياة وتعقدها ظهرت الحاجة إلى استخدام الصور في كثير من المجالات العلمية كالفضائية

والطبية والهندسية، ولكون هذه الصور تتعرض لكثير من التشويه أثناء التقاطها نتيجة توفر الكاميرات والجوالات بكثرة وبأيدي أشخاص لا يملكون الخبرة الكافية في كيفية التقاط الصور من ناحية جودة الإضاءة وتوزيعها فأصبح من المهم استخدام أساليب متتوعة لتحسين إظهار هذه الصور بأفضل شكل ومن هذه الأساليب تحسين تباين

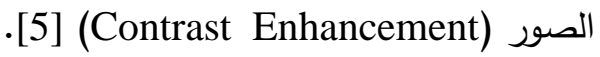

تم في هذا البحث باستخدام برنامج matlab (2008) تطبيق مرشحات خوارزمية تقليدية وهي مرشح تحسين التباين المتكيف العامGlobal Contrast Enhancement Filter (ACE_meanثم تم تطوير هذه Median Contrast ) الخوارزمية إلى خوارزميتين مقترحتين جديدتين هما خوارزمية تحسين التباين الوسيط 
Max \& Min Contrast ) والأخرى خوارزمية تحسين تباين القيمة الكبرى والصغرى (Enhancement

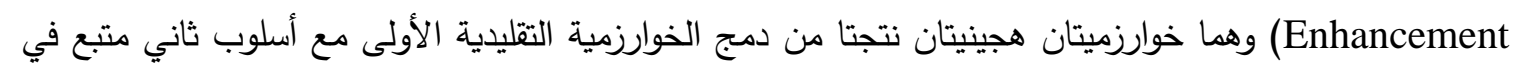

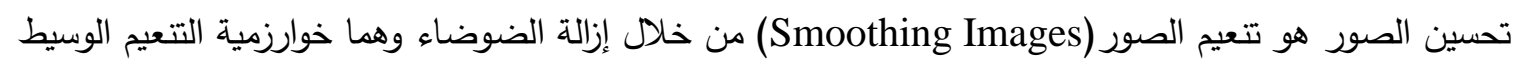
وخوارزمية التتعيم للقيمة الكبرى والصغرى (Median Smoothing)

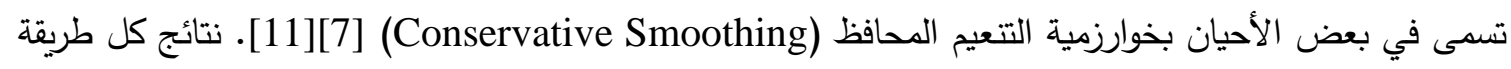
تمت مقارنتها مع النتائج المحصلة من الخوارزميات الأخرى لبيان الأفضل من بينهم وتبين أن الخوارزميتين المقترحتين أفضل من ناحية تحسين تباين الإضاءة من نتائج الخوارزمية التقليدية. الكلمات المفتاحية: تباين، مرشح تحسين التباين المتكيف العام.

1

نتيجة التقدم الكبير الذي حصل في الآونة الأخيرة في شتى المجالات فقد لاحظ الجميع الإقبال الثديد على البرامج المتعلقة بالصورة ووضوحها لذلك أصبح الاهتمام بمبادئ معالجة الصور وتحسين ظهورها من الأمور

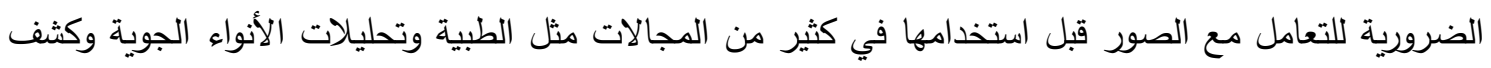
المخالفات المرورية والتعرف على هويات الأشخاص [5]. لذلك تم اختيار مجال البحث للتوصل إلى فئل خوارتميات

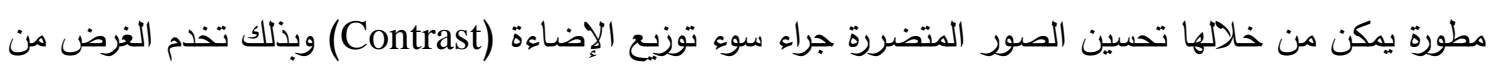

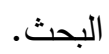

2

يهدف هذا البحث إلى تطبيق خوارزمية التباين عمليا على الصور الرقمية الملونة واستتتاج خوارزميات

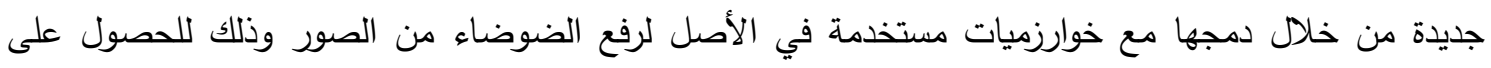
خوارزميات جديدة تستخدم لغرض تحسين نسبة التباين للصور مما يجعل الصور تبدو بشكل أفضل وأوضح من فن لاهن ناحية توزيع إضاءتها. 3

بعد ازدياد الاهتمام بالصور والتعامل معها ازدادت الدراسات التي تهتم بالعمليات التي تتم على الصور

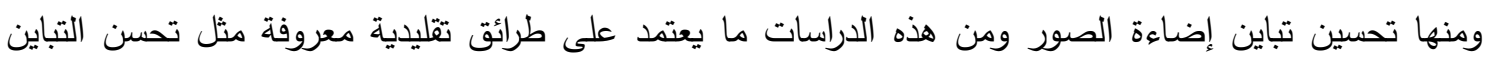

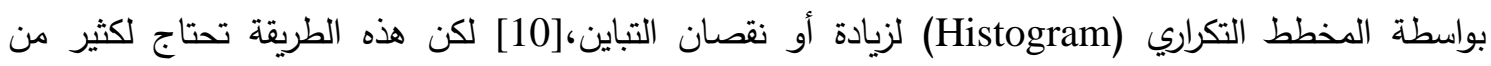
الخطوات كعمل جدول يمثل قيم المخطط التكراري للصورة ثم إيجاد القيم الأفضل لتحسين التباين ومن ثم تحديث

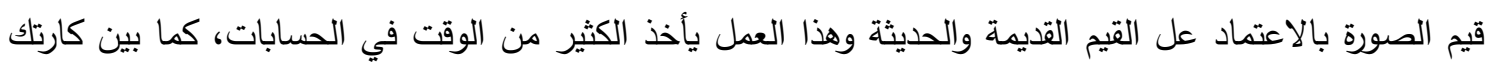

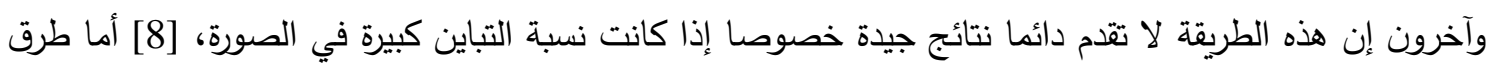
التباين الهحلية (Local Contrast) فمغظمها يعتمد على تجزئة الصور إلى مقاطع (segment) والعمل عليها وهذا أيضا يستغرق وقتاً اكبر من الطريقة العامة (Global Contrast) المتبعة في هذا البحث. كما اعتمد الباحثون على التخمين لإيجاد معدل التباين وهذا الأسلوب قد لا يحسن تباين الصور في بعض الأحيان،[8]. 
وهي عملية معالجة تجرى على بيانات الصور الرقمية يتم فيها تحسين البيانات واستبدالها ببيانات جديدة تصبح فيها الصورة أكثر وضوحا مما يسهل عملية تفسير محتويات الصورة والتعرف على الأهداف التي تغطيها بدقة اكبر • وتتضمن هذه العملية تقنيات تهدف إلى زيادة الفوارق البصرية بين المعالم في الصورة. ومن هذه التقنيات التحسين الإشعاعي للصورة بتقنيات تمديد التباين وتحسين الصورة المكاني باستخدام تقنيات الترشيح والتحسين

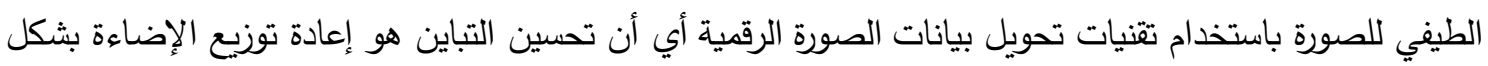

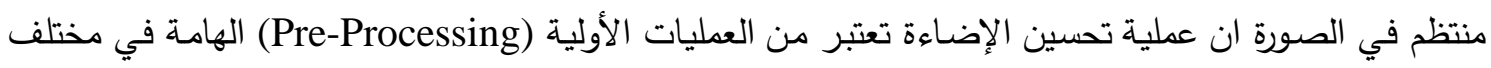
تطبيقات معالجة الصورة إذ قد تكون الإضـاءة سيئة عند التقاط الصورة فلا تظهر الملامح بثكل جيد الإندة وكذلك في

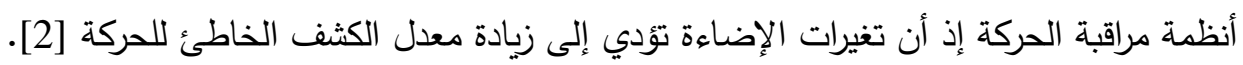

$$
5
$$

تم في هذا البحث إتباع طرائق لتحسين تباين الصور تعتمد على تقنية اعتماد نقاط الصورة كلها في

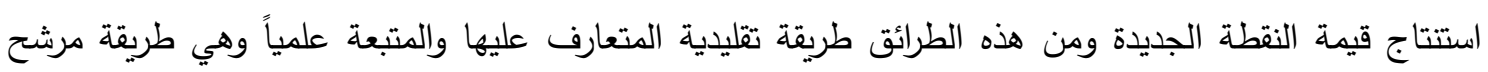

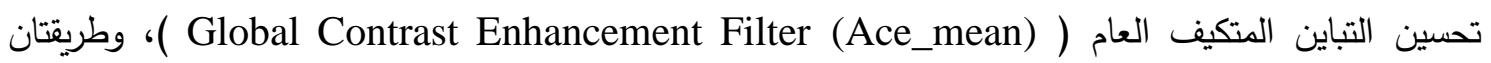

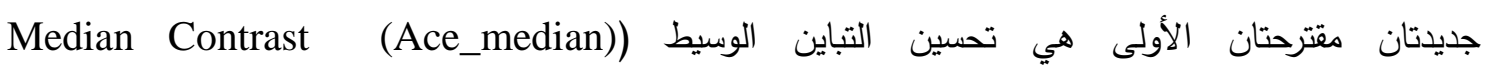
Max \& Min (Ace_maxmin) وطريقة ثانية هي تحسين تباين القيمة الكبرى والصغرى التيطين (Enhancement

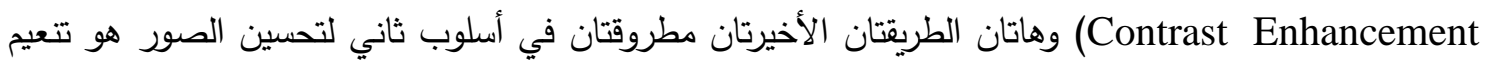

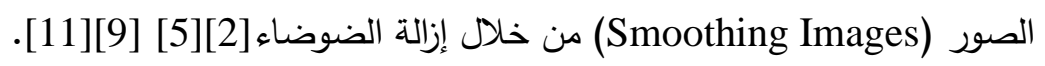

\section{Ace_mean 5.1}

هي اختصار لـ (Global Adaptive Contrast Enhancement ) وهي من الطرائق التقليدية التي تعتمد على متغيرات عامة يتم حسابها ومن ثم تطبيقها على جميع نقاط الصورة كمصفوفة واحدة ولا يتم تجزئة الصورة إلى مقاطع (window) هذه المتغيرات هي حساب المعدل العام للصورة (mean) ثم استخدام هذه القيمة

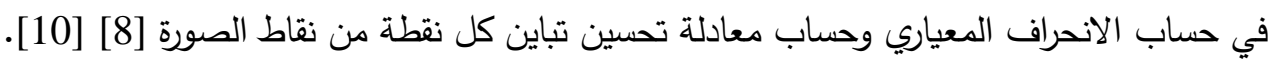

\section{2 طريقة تحسين التباين الوسيط Ace_median}

وهي طريقة هجينة مستتبطة من إحدى طرق إزالة الضوضـاء ( noise enhancement) وهي طريقة المعدل الوسيط (median) [4][5][7] [11] مع طريقة تحسين التباين المتكيف العام السابقة إذ تتثابه خطوات

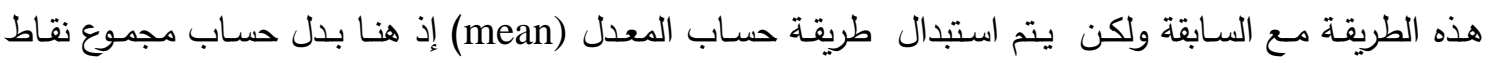

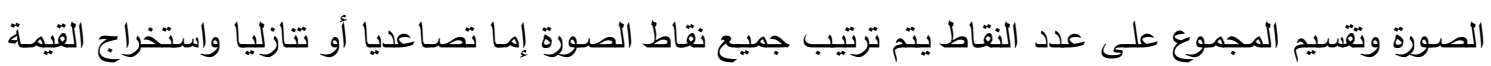
الأوسطية (median) منهم ثم تحوير معادلات تحسين التباين بان يستبدل قيمة المعدل بهذه القيمة الأوسطية.

Ace_maxmin 5.3

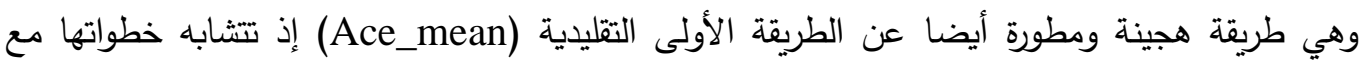
خطوات الطريقة الأولى باستثناء طريقة حساب المعدل (mean) إذ هنا يتم إيجاد اكبر قيمة من بين نقاط الصورة

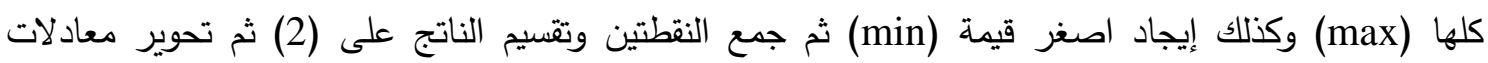
تحسين التباين بأن يستبدل قيمة المعدل العام (mean) بقيمة معدل النقطتين المحسوبة وهذه الطريقة هي إحدى 
طرق إزالة الضوضاء (noise enhancement) [5][7] والتي تسمى بخوارزمية التتعيم للقيمة الكبرى والصغرى وبذلك تكون هذه الطريقة هجينة بين الأسلوبين. (Max \& Min Smoothing)

6

\section{Ace_mean خوارزمية تحسين التباين المتكيف العام 6.1}

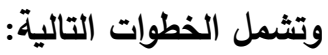

1. قراءة الصورة ذات الإضـاءة السيئة ومـن ثم حسـاب المعدل العـام للصسورة (mean) وذلك بجمع كل نقاط

$$
\text { mean }=\frac{1}{m \times n} \sum \mathrm{i}(\mathrm{m}, \mathrm{n})
$$

الصورة وتقسيم الناتج على حجم الصورة وذلك حسب المعادلة التالية [3]:

2. حساب الانحراف المعياري ( standard deviation ) للصورة وذلك بإيجاد فارق كل نقطة من نقاط الصورة

مع قيمة المعدل العام للصورة والمحسوب في معادلة رقم (1) ثم جمع كل النتائج بعد تربيع هذا الفارق ومن فئن

$$
\begin{aligned}
& \text { ثم تقسيمه على حجم الصورة ثم حساب قيمة الجذر ألتربيعي وذلك حسب المعادلة الآتية [10]: } \\
& \mathrm{Sd}=\sqrt{\frac{1}{m \times n-1} \sum(\mathrm{i}(\mathrm{m}, \mathrm{n})-\text { mean })^{2}}
\end{aligned}
$$

3. حساب معادلة تحسين التباين (Ace) لكل نقطة من نقاط الصورة ومن ثم عرض الصورة الناتجة بعد تطبيق

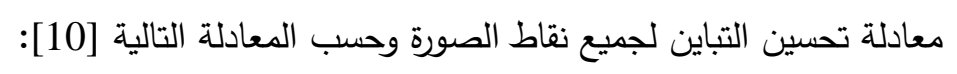

$$
\text { Ace }(m, n)=k 1\left[\frac{\text { mean }}{\text { sd }}\right][i(m, n)-\text { mean }]+\mathrm{k} 2 * \text { mean }
$$

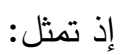

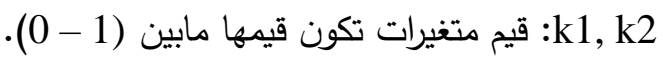

$$
\begin{aligned}
& \text { :m, n }
\end{aligned}
$$

Ace_median 6.2

وتثمل الخطوات التالية: - التمات

1. قراءة الصورة ذات الإضلاءة السيئة ومن ثم استخراج القيمة الوسطية من بين جميع نقاط الصورة وذلك بعمل تصل

ترتيب تصاعدي (sorting) لجميع نقاط الصورة ثم اخذ القيمة الوسطية من بينها.

2. حساب الانحراف المعياري (sd) للصورة لكن بدل استخدام قيمة المعدل mean يتم استخدام القيمة الأوسطية median

$$
\mathrm{Sd}=\sqrt{\frac{1}{\mathrm{~m} \times \mathrm{n}-1} \sum(\mathrm{i}(\mathrm{m}, \mathrm{n})-\mathrm{median})^{2}}
$$

3. حساب معادلة تحسين التباين (Ace) لكل نقطة من نقاط الصورة ومن ثم عرض الصورة الناتجة بعد استبدال قيمة المعدل بالقيمة الأوسطية المحصلة من الخطوة الأولى وقيمة الانحراف المعياري الجديدة لتكون المعادلة Ace median $(m, n)=k 1\left[\frac{\text { median }}{s d}\right][i(m, n)-$ median $]+\mathrm{k} 2 *$ median 
Ace_maxmin تحسين تباين القيمة الكبرى والصغرى 6.3

وتثمل الخطوات التالية: - n

1. قراءة الصورة ذات الإضاءة السيئة ومن ثم إيجاد أعلى (max) واقل (min) قيمة من بين قيم نقاط الصورة ثم حساب معدل النقطتين وذلك حسب المعادلة الآتية:

$\operatorname{maxmi}=(\max +\min ) / 2$

2. حساب الانحراف المعياري ( sd ) للصورة لكن بدل استخدام قيمة المعدل mean يتم استخدام القيمة maxmi

$$
\mathrm{Sd}=\sqrt{\frac{1}{m \times n-1} \sum(\mathrm{i}(\mathrm{m}, \mathrm{n})-\mathrm{maxmi})^{2}}
$$

3. حساب معادلة تحسين التباين (Ace) لكل نقطة من نقاط الصورة ومن ثم عرض الصورة الناتجة بعد استبدال قيمة المعدل بالقيمة (maxmi) المحصلة من الخطوة الأولى وقيمة الانحراف المعياري الجديدة من الخطوة

$$
\text { Ace maxmi }(m, n)=k 1\left[\frac{\operatorname{maxmi}}{s d}\right][i(m, n)-\text { maxmi }]+\text { k2 * maxmi }
$$

لقد تم تطبيق الخوارزميات الثلاثة على نوعية الصسور ذات الامتداد (jpg) وبواقع عشرة صسور لكل الكل

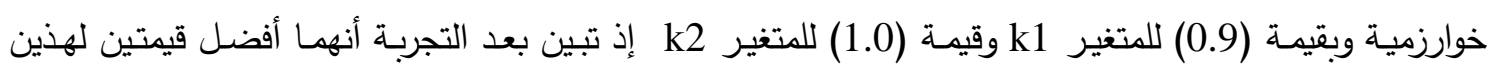
المتغيرين لاحظ الشكل رقم (1).

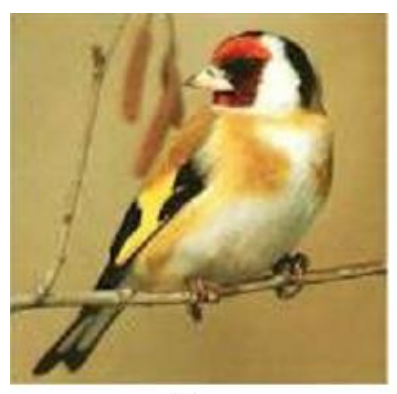

A

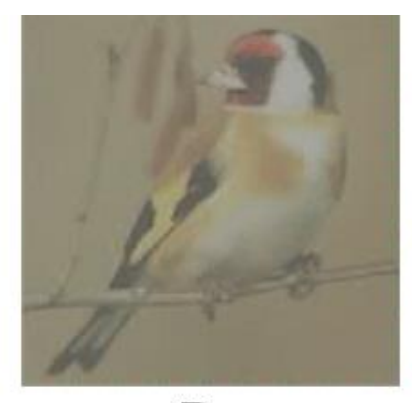

$\mathrm{B}$

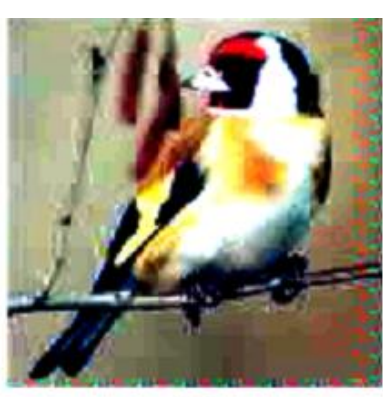

C

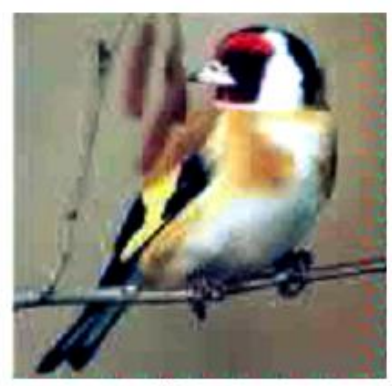

D

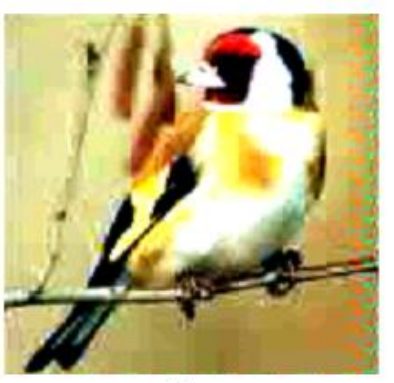

E

شكل (1). نتائج تطبيق خوارزميات تحسين التباين

Ace_mean الصورة الناتجة من تطبيق C الصورة المضببة C الصورة الأصلية A

Ace_maxmin الصورة الناتجة من تطبيق من تطبيق E Ace_median الصورة الناتجة من تطبيق D 
لبيان مدى جودة الصور المحصلة بعد تطبيق خوارزميات تحسين التباين يتم استخدام مقياس مMS

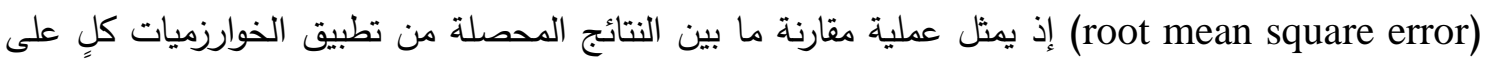
حدة مع الصورة الأصلية ،لاحظ النتائج في الجدول (1). إن معادلة RMS تمثل رياضياً المسافة الإقليدية والتي تجد التباين بين نقاط صورتين قبل المعالجة وبعدها والمعادلة هي [10]:

$$
R M S_{e}=\sqrt{\frac{1}{\mathrm{~m} * \mathrm{n}} \sum_{\mathrm{r}=0}^{m-1} \sum_{\mathrm{c}=0}^{\mathrm{n}-1}\left[\mathrm{I}^{\prime}(\mathrm{r}, \mathrm{c})-\mathrm{I}(\mathrm{r}, \mathrm{c})\right]^{2}}
$$

إذ تمثل 'الصورة الناتجة بعد التطبيق I'، تمثل الصورة الأصلية، m*n حجم الصورة الكلي.

جدول (1). يمثل قيمة الفروقات للصور عند تطبيق الخوارزميات الثلاثة لنوع الصور ذات الامتداد (jpg)

\begin{tabular}{|c|c|c|c|}
\hline خوارزمية تباين القيمة الكبرى & خوارزمية التباين الأوسطي & خوارزمية تحسين التباين & رقم الصورة \\
\hline 68.0274 & 43.4674 & 83.3314 & im1 \\
\hline 40.2021 & 41.4070 & 56.8705 & im2 \\
\hline 46.3344 & 21.0163 & 49.2352 & im3 \\
\hline 57.4020 & 31.3258 & 60.2381 & im4 \\
\hline 28.6881 & 36.3561 & 44.1662 & im5 \\
\hline 51.8338 & 21.4472 & 54.8733 & im6 \\
\hline 44.2332 & 22.8581 & 45.8716 & $\operatorname{im} 7$ \\
\hline 61.8914 & 25.2867 & 66.6571 & im8 \\
\hline 42.6019 & 30.6809 & 44.2675 & im9 \\
\hline 40.2100 & 21.7681 & 32.8488 & im10 \\
\hline
\end{tabular}

وللتأكد من جودة نتائج الصور المحصلة يمكن استخدام مقياس آخر هو (NCC) وهو مقياس معامل

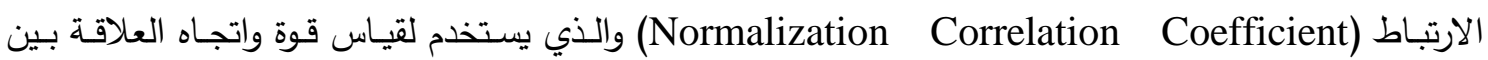
مجموعتين أي بمعنى يقيس العلاقة بين الصورة الأصلية والصورة الناتجة وتشير إثارة القيمة إلى اتجاه الارتباط

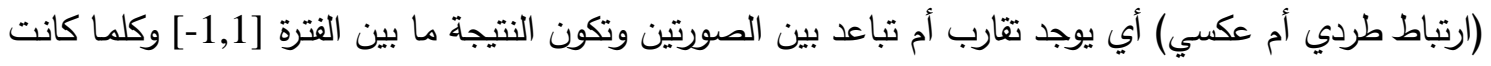

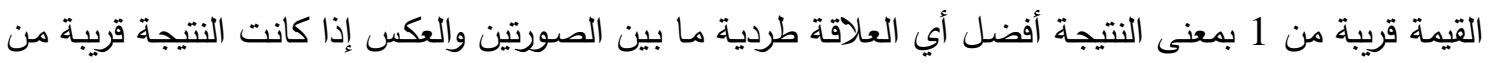
1- أي لا يوجد تقارب والعلاقة عكسية بين الصورتين، لاحظ نتائج الجدول (2). إن معادلة معامل الارتباط هي

$$
N C C=\sum_{x, y} P(x, y) P^{-}(x, y) / \sum_{x, y} P^{2}(x, y)
$$

إذ تمثل p(x,y) النقطة الأصلية، pe (x,y) النقطة المحسنة. 
جدول (2). يمثل قيم مقياس NCC عند تطبيق الخوارزميات الثلاثة لنوع الصور ذات الامتداد (jpg)

\begin{tabular}{|c|c|c|c|}
\hline خوارزمية تباين القيمة الكبرى & خوارزمية التباين الأوسطي & خوارزمية تحسين التباين & رقم الصورة \\
\hline 0.9011 & 0.7874 & 0.7857 & im1 \\
\hline 0.9224 & 0.8407 & 0.7975 & im2 \\
\hline 0.9526 & 0.9447 & 0.9391 & im3 \\
\hline 0.9415 & 0.8839 & 0.8874 & im4 \\
\hline 0.9867 & 0.8968 & 0.8461 & im5 \\
\hline 0.9607 & 0.9361 & 0.9425 & im6 \\
\hline 0.9771 & 0.9477 & 0.9683 & im7 \\
\hline 0.9850 & 0.9688 & 0.9718 & im8 \\
\hline 0.9569 & 0.9368 & 0.9239 & im9 \\
\hline 0.9232 & 0.9347 & 0.9231 & im10 \\
\hline
\end{tabular}

8

تم تطبيق خوارزميات تحسين التباين التقليدية والمطورة على عدد من الصور الملونة ذات أحجام مختلفة وأثكال مختلفة (انظر ملحق A) وتم التوصل إلى الاستنتاجات التالية:

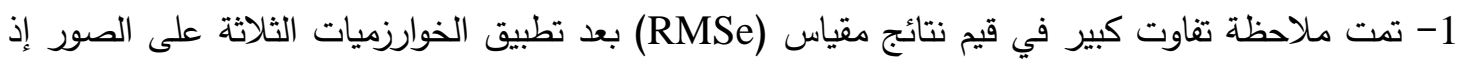
كانت طريقة تحسين التباين الأوسطي أفضل طريقة وذات كفاءة عالية في تحسين تبائ مقياين الصور إذا ما قورنت

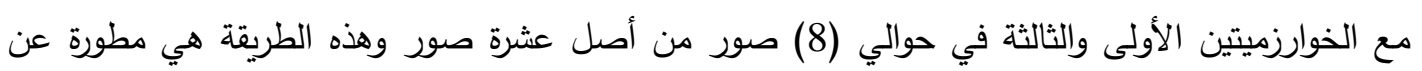

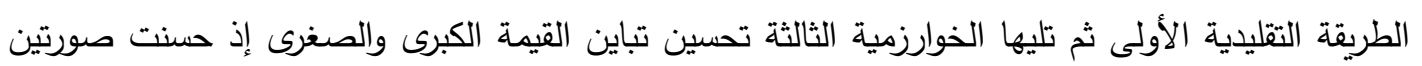

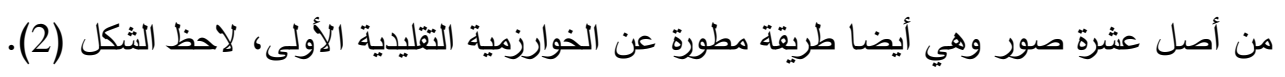
2- عند ملاحظة قيم الجدول رقم (1) نلاحظ انه عندما تكون الصورة محسنة بشكل أفضل بطريقة الخوارزمية المطورة تحسين التباين الأوسطي تكون بفارق كبير عن قيم الطريقتين الأولى والثالثة أي أن تحسينها للصور

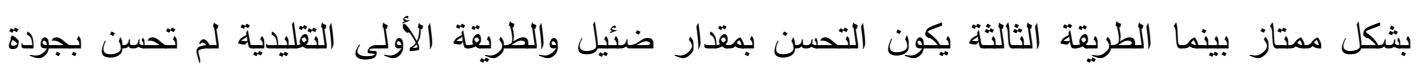
الطريقتين المقترحتين. 3- من ملاحظة قيم الجدول (2) الذي يمثل قيم معامل الارتباط NCC كانت خوارزمية تحسين تباين القيمة الكبرى والصغرى أفضل بحوالي (9) صور من أصل عشرة وصورة واحدة أفضل بطريقة تحسين التباين

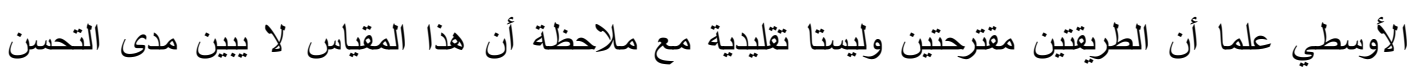

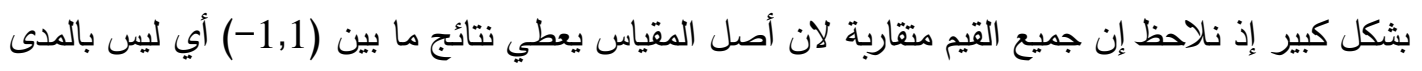

الكبير، لاحظ الثكل (3). 


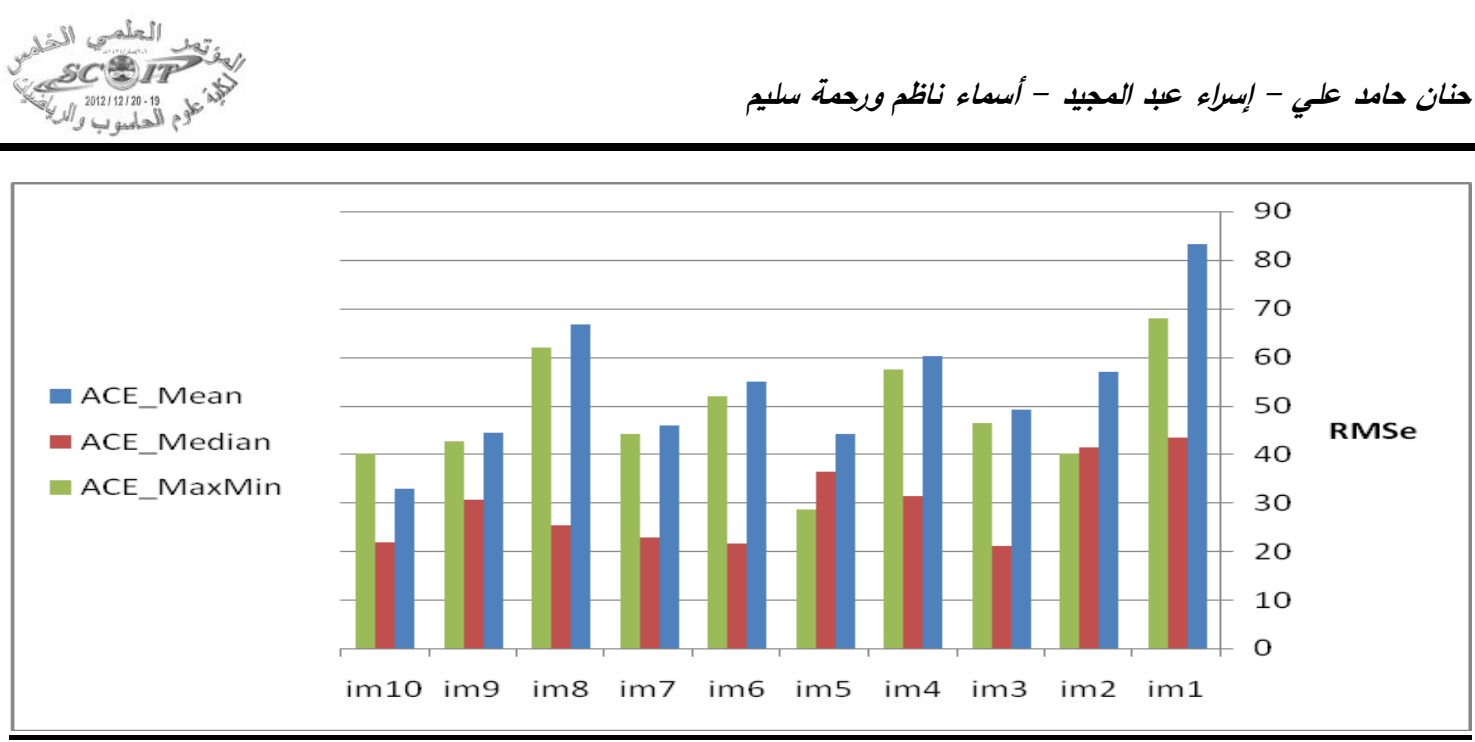

الثكل (2). مخطط بياني يوضح مقدار الاختلاف في تحسين التباين للخوارزميات الثلاثة لمقياس RMSe

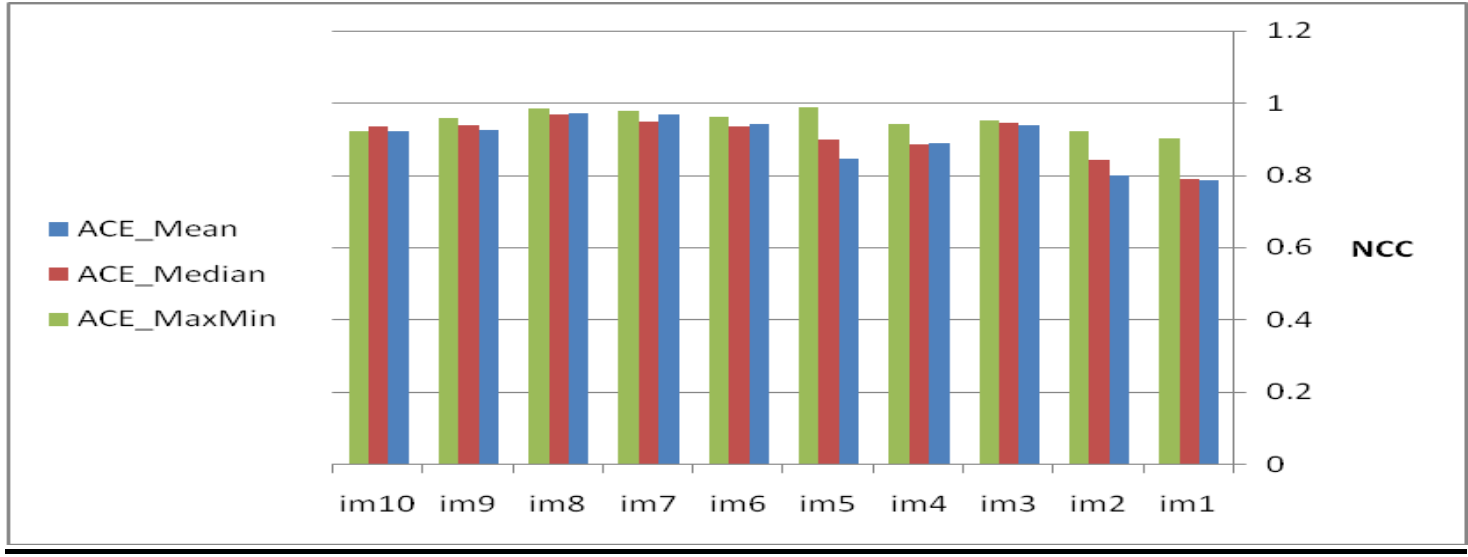

الثكل (3). مخطط بياني يوضح مقدار الاختلاف في تحسين التباين للخوارزميات الثلاثة لمقياس NCC 


\section{المصادر}

الخياط، باسل يونس، (2010)، "مدخل إلى المحاكاة التصادفية الحاسوبية ونمذجتها باستخدام

صـلاحو، محمد نجيب، "طرق تحسين الصـورة الرقمية"، كلية الهندسة الكهربائية والالكترونيـة، جامعـة

حلب.http://www.eng2all.net/vb/t40959.html حلب

علي، حنان حامد، (2008)، "خوارزمية هجينة للتخلص من ضوضاء الصور"، مجلة علوم الرافدين لعلوم

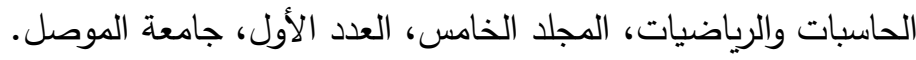

[4] Gomes, J., and Velho L., (1997), "Image Processing for Computer Graphics", Translated by Silvio L., springer, inc.

[5] Gonzalez, Rafael E., (2002), "Digital Image Processing", $2^{\text {nd }}$ edition, publisher: prentice-Hall.

[6] Katzenbeisser, S.,and Petitcolas, F., (1999), "Information Hiding Techniques for Steganography and Digital Watermarking", Artech House, Boston London.

[7] Robert, F.; Simon P., and Walker A., (2003), "Digital Filters" http://homepages.inf.ed.ac.uk/rbf/HIPR2/filtops.htm.

[8] Subr, K.; Majumder, A., and Sandy, I., "Greedy Algorithm for Local Contrast Enhancement of Images " ,University of California.

http://www.springgerlink.com/content/yhu486x7mu124r66.

[9] Tinku, A., and Ajoy, K., (2005), "Image Processing Principles and Application", WILEY - Interscience USA.

[10] Umbaugh, Scott E. ,(1998) ,Computer Vision and Image Processing a Practical Approach using CVIP Tools", Prentice Hall PTR.

[11] William, K., (2007), "Digital Image Processing", Fourth Edition, Wiley \& Sons. Inc. Publication. 


\section{ملحق (A)}

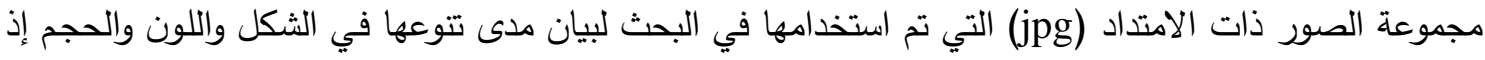
تمثل A الصورة الأصلية ، B الصورة المضببة ، C الصورة الناتجة من تطبيق الخوارزمية الأولى تحسين التباين

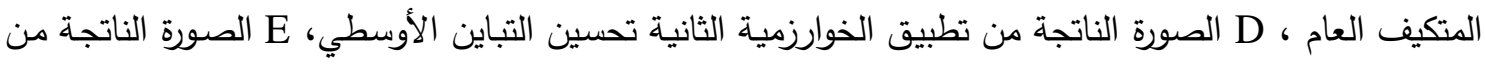
تطبيق الخوارزمية الثالثة تحسين تباين القيمة الكبرى والصغرى.لاحظ أرقام الصور وقيم (RMSe) في الجدول رقم

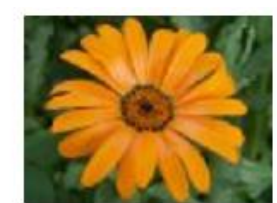

$\operatorname{Im} 2$

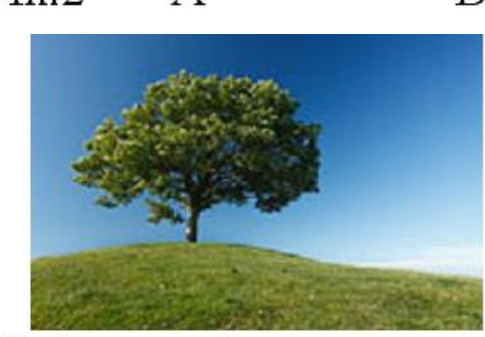

$\operatorname{Im} 3$

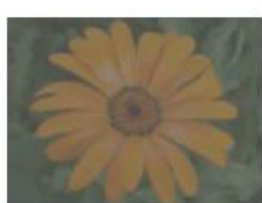

B

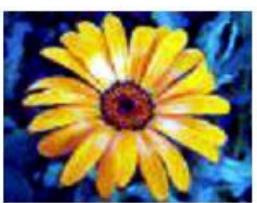

C

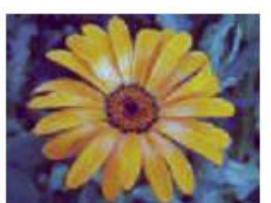

D

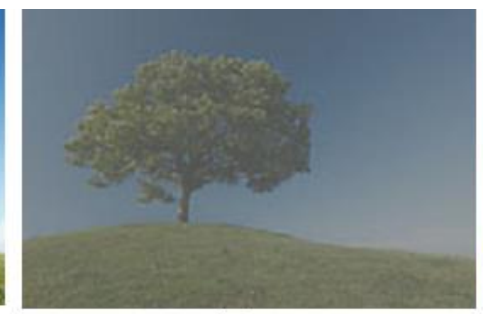

B

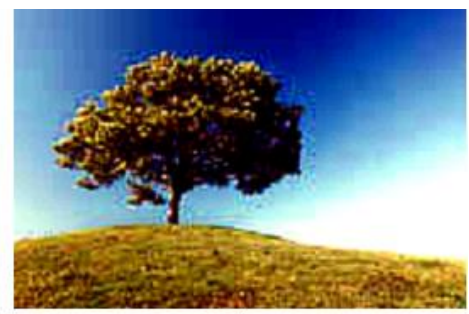

C

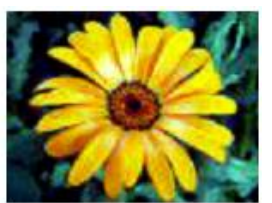

E

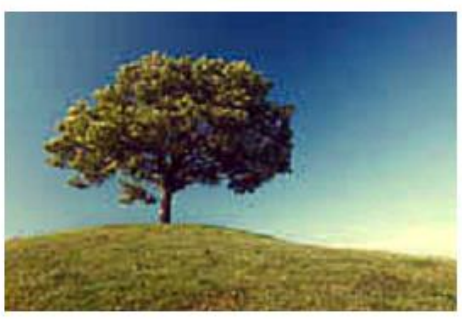

D

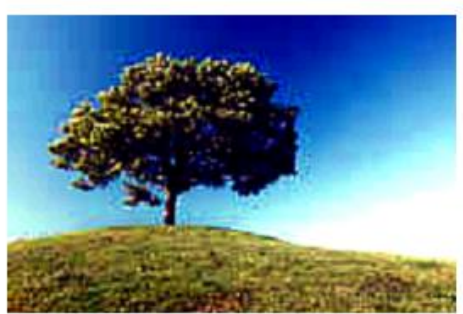

E

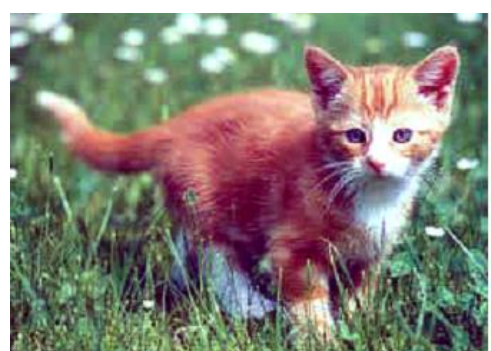

Im 4

A

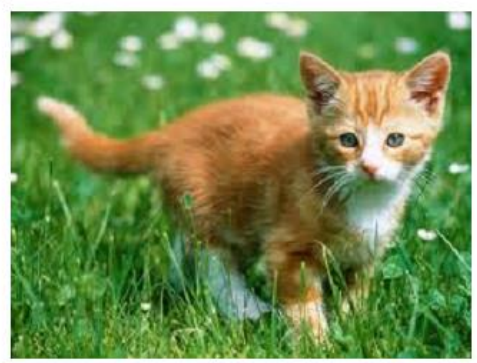

D
B

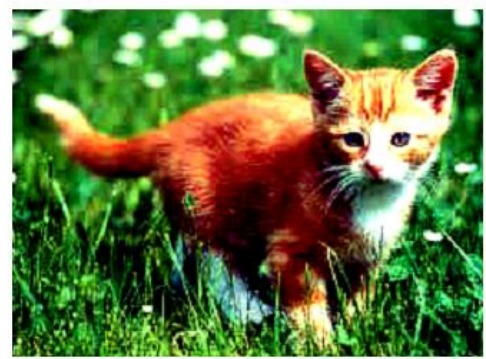

E 


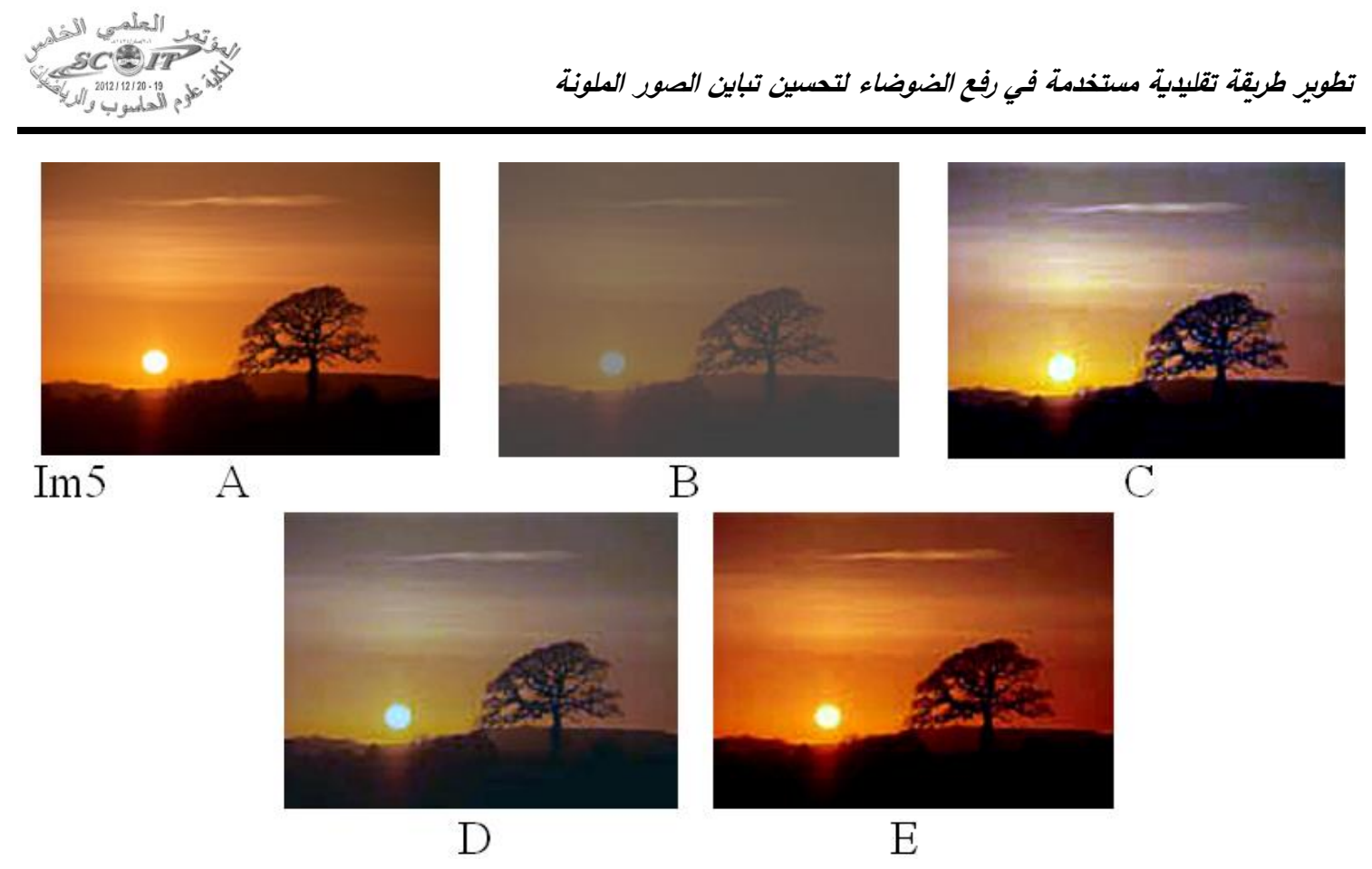

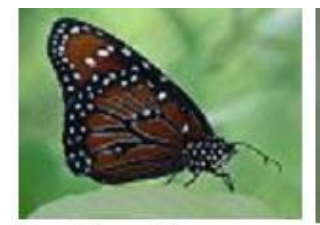

Im6 A

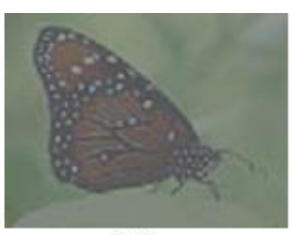

B

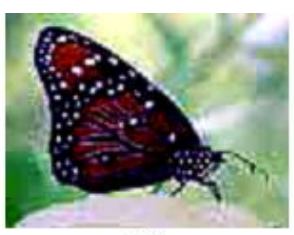

C

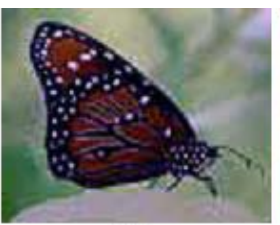

D

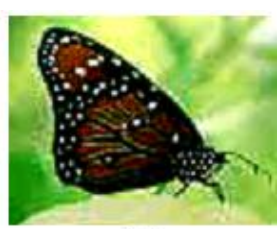

E

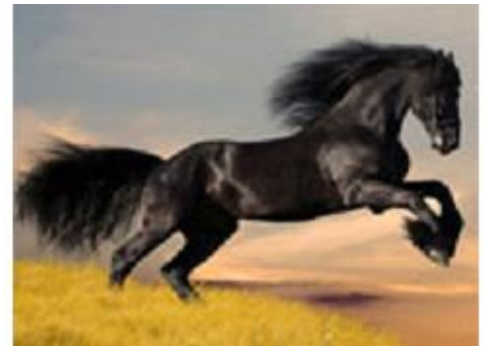

$\operatorname{Im} 7$

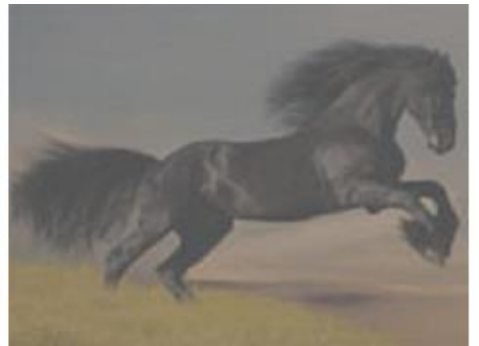

B

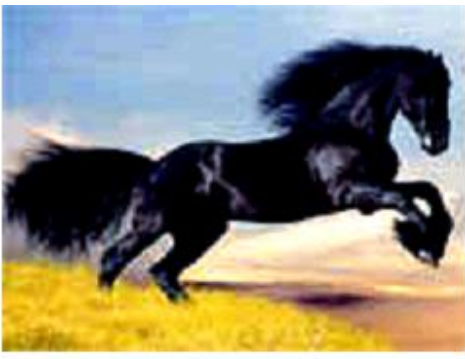

C

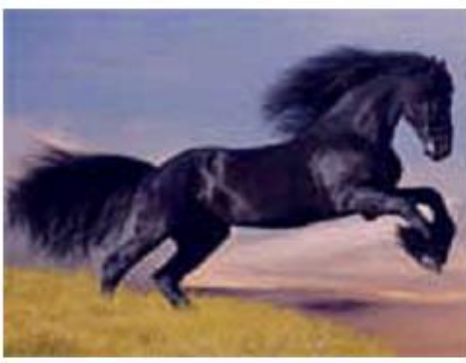

D

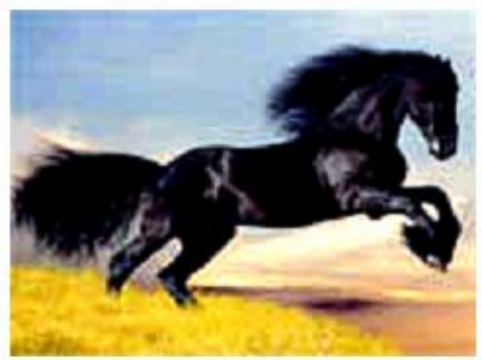

E 

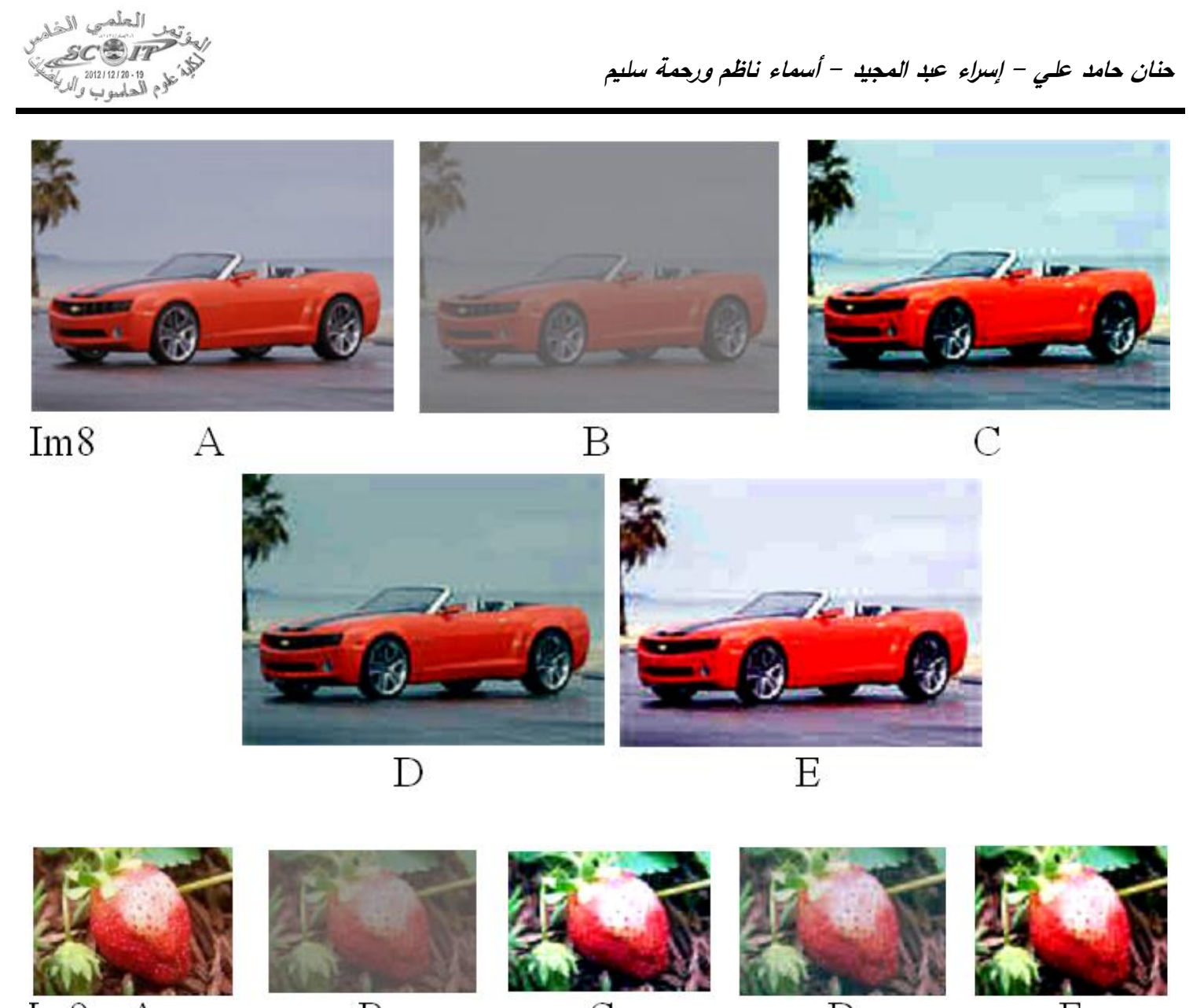

B

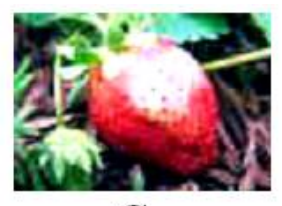

C

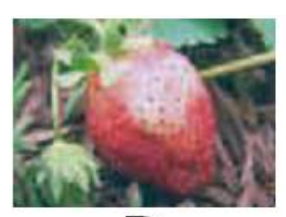

D

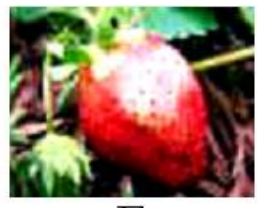

E

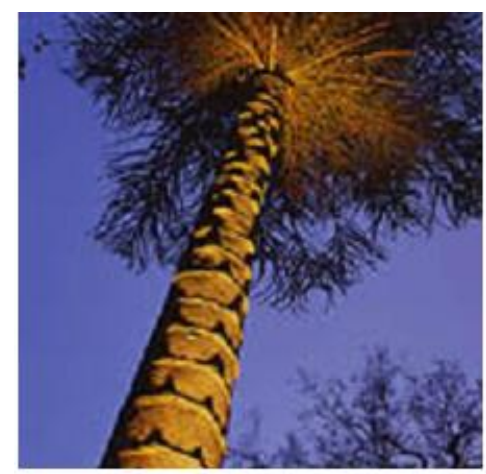

$\operatorname{Im} 10 \mathrm{~A}$

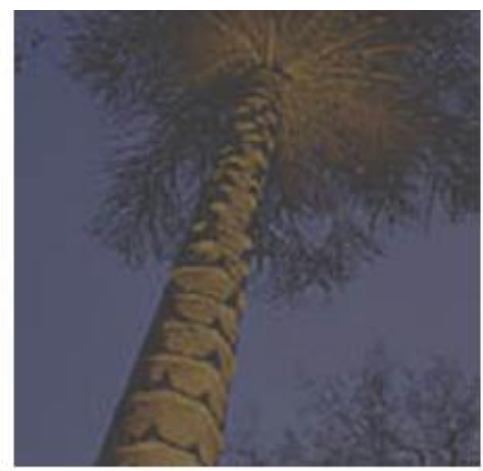

B

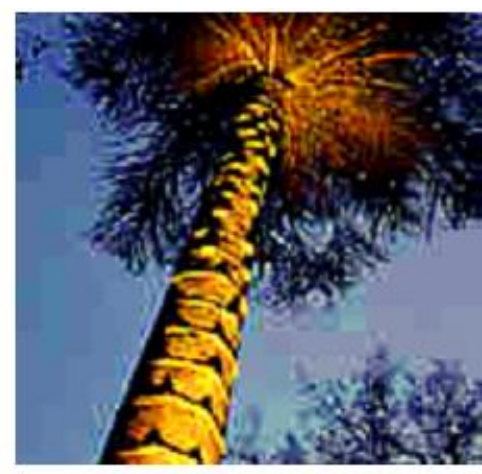

C

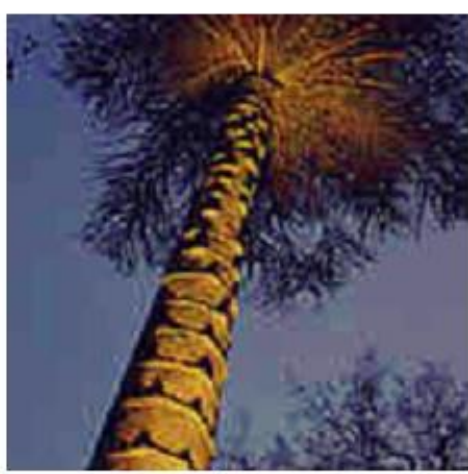

D

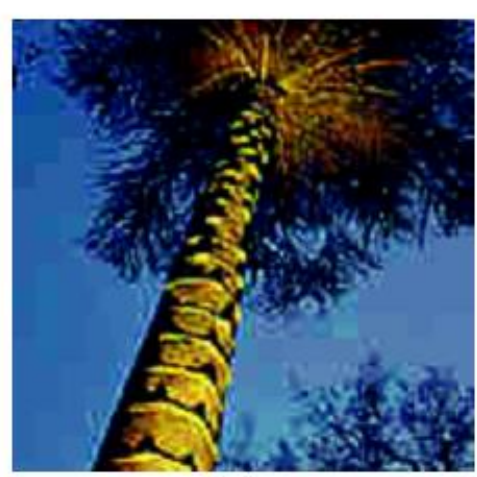

E 